Modern Physics Letters A

(C) World Scientific Publishing Company

\title{
SEARCH FOR ULTRA-HIGH ENERGY PHOTONS USING AIR SHOWERS
}

\author{
MARKUS RISSE \\ University of Wuppertal, Department of Physics \\ Gaußstr. 20, 42097 Wuppertal, Germany \\ risse@physik.uni-wuppertal.de \\ PIOTR HOMOLA \\ H. Niewodniczański Institute of Nuclear Physics PAN \\ ul. Radzikowskiego 152, 31-342 Kraków, Poland \\ piotr.homola@ifj.edu.pl
}

Received (Day Month Year) Revised (Day Month Year)

\begin{abstract}
The observation of photons with energies above $10^{18} \mathrm{eV}$ would open a new window in cosmic-ray research, with possible impact on astrophysics, particle physics, cosmology and fundamental physics. Current and planned air shower experiments, particularly the Pierre Auger Observatory, offer an unprecedented opportunity to search for such photons and to complement efforts of multi-messenger observations of the universe. We summarize motivation, achievements, and prospects of the search for ultra-high energy photons.
\end{abstract}

Keywords: Ultra-high energy photons, cosmic rays, air showers

96.40.Pq,96.40.-z,13.85.-t,13.85.Tp

\section{Introduction}

Photons are the main messenger particles for exploring the universe. Over the last decades, the wavelength range of photon observation was dramatically expanded and stretches now from radiowaves to high-energy gamma rays. The current maximum energy of photons observed is $\sim 10^{14} \mathrm{eV} !$

In this short review, we focus on the search for photons of energies above $10^{18} \mathrm{eV}$ up to the highest energy (few times $10^{20} \mathrm{eV}$ ) by measuring particle cascades (air showers) initiated in the atmosphere of the Earth. An observation of such ultrahigh energy (UHE) photons, several orders of magnitude in excess of currently observed photon energies, would open a new window in cosmic-ray research with significant impacts on related research fields. Giant air shower experiments, most prominently the Pierre Auger Observatory 2 , are unique tools to explore this photon energy range. The expected sensitivity of the Auger Observatory after installation of a large northern array reaches the photon fluxes predicted in more conservative 
scenarios of cosmic-ray origin, making an observation of UHE photons feasible.

UHE photons are also inherently linked to other messenger particles such as charged cosmic rays $3 / 41516$ and neutrinos $7 / 8$. The search for UHE photons complements current experimental efforts towards multi-messenger observations of the universe.

The structure of the paper follows the possible "life" of UHE photons. Production scenarios and propagation of photons as well as their predicted fluxes at Earth are shortly described in Section 2. Geomagnetic and atmospheric cascading and the challenge of identifying photon showers are discussed in Section 3 , Current observational results and future prospects are summarized in Section 4 . Throughout Sections 2-4, conceptual issues important for the data interpretation are pointed out. The possible impact of photon searches and detections on different research fields is briefly outlined in Section 5 . Conclusions are given in Section 6 .

\section{UHE Photons As Cosmic Rays}

Production. Though at different levels, most models predict UHE photons, mainly from the decay of neutral pions produced previously by a "primary process",

$$
\text { primary process } \rightarrow \pi^{0}\left(+\pi^{ \pm}\right)+\ldots \rightarrow \gamma_{\mathrm{UHE}}\left(+\nu_{\mathrm{UHE}}\right)+\ldots
$$

In non-acceleration model $\$ 9$, the primary process is given by the decay or annihilation of primordial relics such as topological defects10111 (TD) or super heavy dark matter 12[13]14|15] (SHDM). From considerations of QCD fragmentation, 16 17]18|19 copious photons are then expected to be produced!20|21|22 In the Z-burst scenarid $23|24| 25$ (ZB), photons are generated via the resonant production of $\mathrm{Z}$ bosons by UHE neutrinos annihilating on the relic neutrino background.

In more "conventional" cosmic-ray models, nuclear primaries are accelerated at suitable astrophysical sites to ultra-high energy. UHE photons can be produced during propagation by the GZK-type process $26 / 27$ of resonant photo-pion production of UHE nucleons with the cosmic microwave background. The energies of these "GZK photons" are typically a factor $\sim 10$ below the primary nucleon energy. An enhanced production of photons $>10^{18} \mathrm{eV}$ can occur from nuclear primaries passing near the galactic center region. 28

Propagation. UHE photons can initiate electromagnetic cascades by interacting with background radiation fields,

$$
\gamma_{\mathrm{UHE}}+\gamma_{\text {background }} \rightarrow e^{ \pm} \rightarrow \ldots \rightarrow \gamma_{\mathrm{GeV}-\mathrm{TeV}}+\ldots
$$

Significant uncertainties exist for the low-frequency (few $\mathrm{MHz}$ ) radio background and (for $e^{ \pm}$propagation) extragalactic magnetic fields. 2021 Typical energy loss lengths assumed for UHE photons range between $7-15 \mathrm{Mpc}$ at $10^{19} \mathrm{eV}$ and 5$30 \mathrm{Mpc}$ at $10^{20} \mathrm{eV}$. In SHDM models, the relic particles are clustered as cold dark matter in our Galaxy, and UHE photons, as their decay products, would be observed 


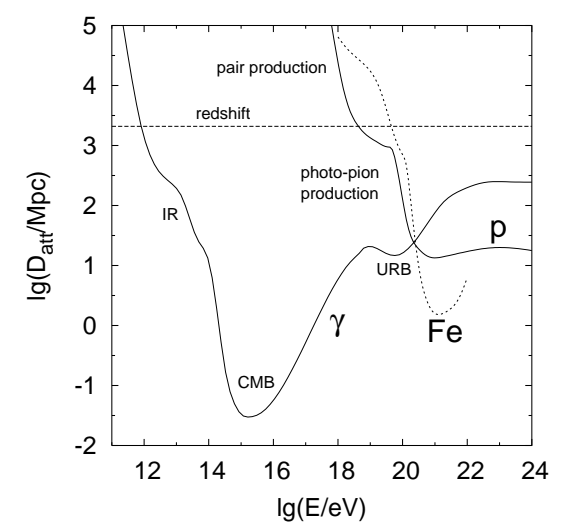

Fig. 1. Energy loss length of photons for interactions with infrared (IR), cosmic microwave (CMB) and universal radio (URB) backgrounds. Uncertainties exist for the URB (see text) and IR background. For comparison, curves for protons and iron nuclei are added. Values are compiled from Refs 929130.

at Earth with little processing. In TD and ZB models, UHE photons are injected at larger distance from the Earth, and part of these photons can cascade to lower energy. The electromagnetic cascade stops at $\mathrm{GeV}-\mathrm{TeV}$ energies where the universe becomes increasingly transparent for photons, see Figure 1

Flux predictions. In addition to the mentioned uncertainties of UHE photon propagation, there are free parameters within the theoretical source models such as density and lifetime of primordial relics in non-acceleration models, injection spectrum in acceleration models, source distribution, etc. To calculate the (range of) photon fluxes predicted by a model, the measured cosmic-ray spectrum is usually taken as a constraint. The question arises which spectrum to use. Firstly, the absolute UHE cosmic-ray flux differs between the experiments by a factor $\sim 2$. This problem may be reduced by regarding the fraction of photons in the cosmic-ray flux rather than absolute rates. Secondly, it is unclear so far whether a suppression of the flux ("GZK cutoff", 26|27) above $E_{\mathrm{GZK}} \sim 6 \times 10^{19} \mathrm{eV}$ exists (as indicated by HiRes data 31 ) or not (as indicated by AGASA data 32 ). The shape of the assumed energy spectrum can affect also the predicted fraction of photons.21/33

Non-acceleration models are usually calculated assuming a spectrum without flux suppression. These models aim at explaining the highest-energy end of the spectrum only $\left[33\right.$ For energies up to $\sim E_{\mathrm{GZK}}$, a "conventional" component of nuclear primaries must be assumed. Then, a common prediction of the models is a dominant photon component at $\sim 10^{20} \mathrm{eV}$, see Figure 2. A reduction of the predicted photon fraction may formally be achieved by introducing a flux suppression above $E_{\mathrm{GZK}}$ which increases the relative importance of the "conventional" nuclear component. However, at the same time this reduces the relevance of non-acceleration models to explain any observed events at all.

For acceleration models, in turn, it seems more natural to assume a spectrum with flux suppression as no nearby astrophysical sources could be identified by now. The predicted photon fluxes are typically relatively small. Fractions of order $\sim 0.1 \%$ 


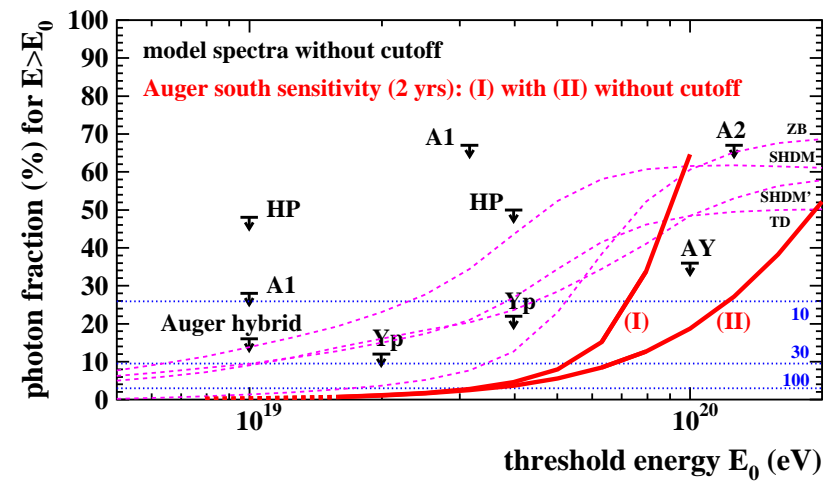

Fig. 2. Fraction of photons in the integral cosmic-ray flux as a function of the threshold energy. The predictions of non-acceleration models (dashed lines: ZB, SHDM, TD from 21, SHDM' from ${ }^{22}$ ) follow a spectrum without flux suppression above $E_{\mathrm{GZK}}$. Experimental limits (Section 4.1 all limits at $95 \%$ c.l.) are reported from Auger hybrid observations (Auger hybrid) 34 , Haverah Park measurements (HP) 35|36, AGASA data (A1) 37], (A2) 38, Yakutsk data (Yp, preliminary) 39, and a combination of AGASA and Yakutsk data (AY) 40 T1. To illustrate the minimum number of events required to place a (95\% c.l.) limit to a certain photon fraction, horizontal dotted lines are shown with the number of events assigned. An estimate (Section 4.2) of the sensitivity of the southern Auger Observatory for two years of operation is given for the two cases that the real spectrum (I) does have, (II) does not have a cutoff. The data from the southern Auger Observatory allow a stringent test of non-acceleration scenarios even if the real spectrum had a cutoff.

were obtained in scenarios assuming nucleon sources,, 2142 with a considerable range of uncertainty (see Figure 2). Larger photon fractions may emerge for specific assumptions on source features, particularly when trying to reproduce a spectrum without flux suppression.21 In case of primary nuclei, the rate of GZK produced photons may be suppressed due to the higher total energy of the nucleus required. Further investigations of such scenarios and of the relation between the photon flux and model parameters are desirable.

A caveat seems in place. The reconstruction of the energy spectrum from shower observations itself requires, to some extent, the fraction of photons as an input (Section 3.3). Usually, no contribution from primary photons is assumed in the reconstruction. For model predictions, a self-consistent comparison to data is required.

\section{UHE Photons as Air Shower Primaries}

At the Earth, photons initiate almost purely electromagnetic showers via pair production and bremsstrahlung. Additional processes are important at highest energy.

\subsection{Specific high-energy processes}

Preshower effect. Contrary to nuclear primaries, $\sim 10^{20} \mathrm{eV}$ photons can convert in the geomagnetic field to an $e^{ \pm}$pair which then emits synchrotron 

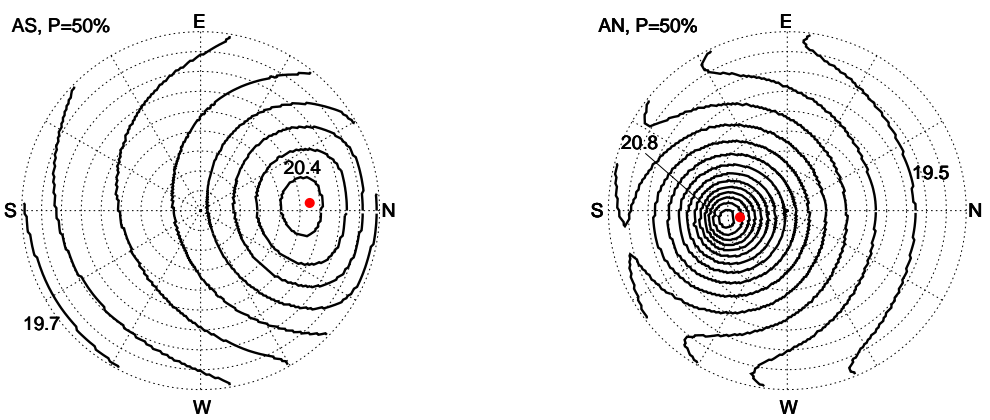

Fig. 3. Sky maps of photon energies at which the conversion probability is $P_{\text {conv }}=50 \%$ for the southern (left) and planned northern (right) Auger sites $[50]$ Contour lines are given with a stepsize of $\Delta \lg (E / \mathrm{eV})=0.1$, minimum and maximum energies are assigned. Azimuthal directions are labeled ("E" for East etc.). Zenith angles are given as concentric circles in $10^{\circ}$ steps (zenith in the center). With increasing energy, the sky fraction grows where the preshower process is important. At Auger North, preshowering starts at factor $\sim 2$ smaller energy compared to Auger South due to the stronger local magnetic field. Close to the pointing direction of the local magnetic field (indicated by a red dot), the highest energies are required for conversion to occur. Accordingly, the sky patterns are shifted in local coordinates between the sites. Despite the larger magnetic field at Auger North, higher energies are needed to reach $P_{\text {conv }}=50 \%$ close to the local field direction. This is connected to the field lines being less curved with altitude at Auger North. The differences between the site characteristics can be exploited in photon searches 50

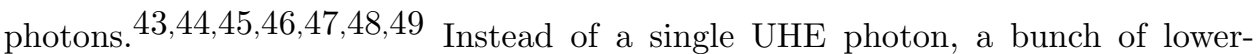
energy electromagnetic particles, called "preshower", enters the atmosphere with important consequences for the shower development. The local differential probability of photon conversion as well as the probability distribution of synchrotron photons emitted by the electrons, depend on the parameter

$$
\chi=\frac{E}{m c^{2}} \frac{B_{\perp}}{B_{\mathrm{c}}}, \quad B_{\mathrm{c}} \sim 4.414 \times 10^{13} \mathrm{G}
$$

where $E$ is the energy of the parent particle (photon or electron), $m$ the electron mass, $B_{\mathrm{c}}$ a constant, and $B_{\perp}$ is the local magnetic field component transverse to the direction of the particle's motion. Since $B_{\perp}$ is involved, preshower characteristics depend strongly on the specific trajectory through the magnetosphere and, thus, on the arrival direction and the experimental site (see Figure 3). The probability $P_{\text {conv }}$ of a photon to convert in the Earth's magnetic field results from an integration along the particle trajectory; for $P_{\text {conv }} \rightarrow 1$, photons would almost surely undergo geomagnetic cascading. Non-negligible probabilities $\left(P_{\text {conv }} \sim 10 \%\right)$ are usually obtained if values $\chi>0.5$ are reached along the trajectory, corresponding to photon energies above $2-4 \times 10^{19} \mathrm{eV}$ depending on the site.

The energy partition in the produced $e^{ \pm}$pair is usually symmetric (ratio of energies $<2$ ), with the probability of asymmetric share increasing with $\chi \underline{49151}$ The spectrum of synchrotron photons becomes harder for larger values of $\chi$. Secondary photons of sufficiently high energy can convert again. 
A typical preshower from a $10^{20} \mathrm{eV}$ photon starts at $\sim 1000 \mathrm{~km}$ altitude and enters the atmosphere $\left(\sim 100 \mathrm{~km}\right.$ altitude) with one or a few $e^{ \pm}$pairs around $10^{18} \mathrm{eV}$ and a large number $(\sim 500)$ of photons. The photon energies extend over several decades, with a few photons around $10^{19} \mathrm{eV}$. Significant fluctuations around these averages may occur, particularly when the conversion takes place at low altitudes. The spread of preshower particles in transverse distance and arrival time is well below current detector resolutions; the subsequent air shower is observed as one event. 52

To simulate the preshower effect, Monte Carlo codes have been developed. The PRESHOWER ${ }^{52}$ program is available as a standalone tool and is also linked to the CORSIKA $^{53}$ and CONEX ${ }^{54}$ shower codes. An independently developed preshower program (MaGICS55) is available within the shower code AIRES 56 . A realistic model of the geomagnetic field such as the IGRF 57 model, is an essential ingredient of precise calculations. As an example, a simple dipole model fails to reproduce the factor $\sim 2$ difference in local field strength at the southern and northern Auger sites.

LPM effect. In a medium, the Bethe-Heitler cross-section 58 for pair production by photons $\left(\sigma_{\mathrm{BH}} \approx 0.51 \mathrm{~b}\right.$ in air 59 ) can be reduced due to destructive interference from several scattering centers. This so-called LPM effect (Landau and Pomeranchuk $\frac{6061}{6}$, Migda $[62$ ) is confirmed by experiments, see Refs $\underline{6364}$ for reviews. With

$$
\kappa=\frac{E_{\gamma} E_{L P M}}{E_{e}\left(E_{\gamma}-E_{e}\right)}, \quad E_{\mathrm{LPM}}=\frac{m^{2} c^{3} \alpha X_{0}}{4 \pi \hbar \rho} \approx(7.7 \mathrm{TeV} / \mathrm{cm}) \times \frac{X_{0}}{\rho},
$$

the reduced cross-section $\sigma_{L P M}$ can for $\kappa<1$ be approximated 6364 by $\sigma_{L P M}=$ $\sigma_{B H} \sqrt{\kappa} \propto\left(\rho E_{\gamma}\right)^{-\frac{1}{2}}$, with photon energy $E_{\gamma}$, electron energy $E_{e}$, radiation length $X_{0} \sim 37 \mathrm{~g} \mathrm{~cm}^{-2}$ in air, density $\rho$ of the medium, and electron mass $m$. It follows from Eq. (44) that the reduction is largest for conversion to a symmetric electron pair $\left(E_{e} \approx E_{\gamma} / 2\right)$. The cross-section for producing a highly asymmetric pair $\left(E_{e} / E_{\gamma} \rightarrow 0\right.$ or 1) changes only slightly. In a similar way, also bremsstrahlung is suppressed $59 \mid 65$ Numerical examples (see e.g. Ref. 66 ) are $E_{\mathrm{LPM}} \sim 2.8 \times 10^{17} \mathrm{eV}$ at $300 \mathrm{~m}$ a.s.l. and $\sim 10^{19} \mathrm{eV}$ in the upper atmosphere.

The LPM effect delays the development of an air shower initiated by a single UHE photon as those processes are suppressed that degrade the energy carried by individual high-energy particles. Fluctuations can be very large due to a positive correlation of the reduction of $\sigma_{L P M}$ since $\sigma_{L P M}\left(X_{2}\right)<\sigma_{L P M}\left(X_{1}\right)$ for depths $X_{2}>X_{1}$. The LPM effect is accounted for in AIRES, CORSIKA, and CONEX.

Photonuclear interactions. Photon-initiated cascades are almost purely electromagnetic ones. Production of muon pairs is suppressed by $\left(m_{e} / m_{\mu}\right)^{2}$. The crosssection for photonuclear interactions which mainly transfer energy to secondary hadrons (and these subsequently to muons), is expected to be $\sim 10 \mathrm{mb}$ at $10^{19} \mathrm{eV}$ and thus more than two orders of magnitude below $\sigma_{\mathrm{BH}}$ (which, however, can be 


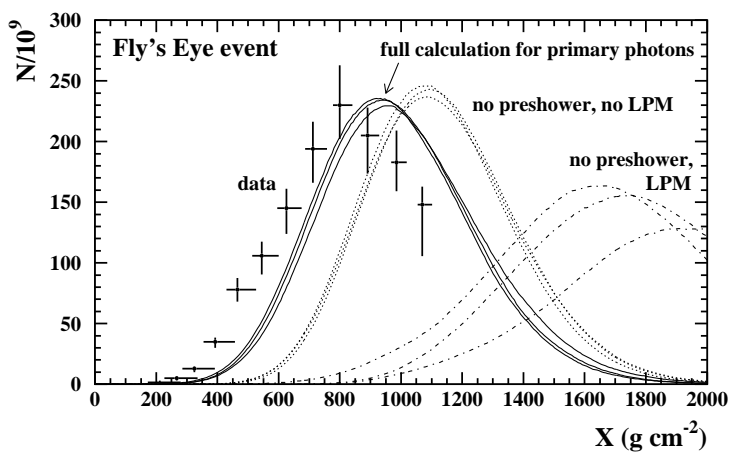

Fig. 4. Observed profile of the $3.2 \times 10^{20} \mathrm{eV}$ Fly's Eye event 75 (data points) compared to simulations 76 assuming primary photons when neglecting both preshower and LPM effect (dotted line), accounting for LPM effect only (dotted-dashed line), and accounting for both effects (solid line). Data points are correlated in $X$. Photons differ by $\sim 1.5 \sigma$ from the data which corresponds to a $\sim 13 \%$ chance probability $76 \mid 77$

reduced by the LPM effect).

One important consequence is that our lack of knowledge of hadron dynamics at high-energy - a major limitation for conclusions about the nuclear composition is of much smaller impact when calculating photon showers. For instance, depths of shower maxima differ by $30-40 \mathrm{~g} \mathrm{~cm}^{-2}$ between SIBYLL 67 and QGSJET 68169 for UHE protons (see Section 3.2, Figure 5) but by less than $\sim 5 \mathrm{~g} \mathrm{~cm}^{-2}$ for UHE photons 34 . For photon searches, data can be compared to photon simulations only, without subtracting a background from nuclear primaries.

As another consequence, certain photon shower observables, in particular the number of secondary muons, are sensitive to UHE extrapolations of the photonuclear cross-section. Different extrapolations of the photon-proton cross-section exist in the literature, see e.g. Refs ${ }^{70 / 71}$ for compilations. For UHE photon searches, it is important to know whether the cross-section could grossly exceed an extrapolation such as provided by the Particle Data Group (PDG), mean photon showers were actually more similar to those of nuclear primaries; an absence of UHE photons may then erroneously be concluded from data. Using a dipole formalism and taking unitarity constraints into account, values of the UHE cross-section that exceed the PDG extrapolation by more than $\sim 80 \%$ (less at smaller energy) were found to be disfavored ${ }^{71}$ It should be noted that for shower simulations, finally the photon-air cross-section is needed, which requires an account for nuclear effects (see e.g. Ref. $\stackrel{71}{ }$ ). From simulations with modified cross-sections, uncertainties for photon showers of $\sim 10 \mathrm{~g} \mathrm{~cm}^{-2}$ in $X_{\max }$ (depth of shower maximum) and $\sim 15 \%$ in $N_{\mu}$ (number of muons at ground) were estimated 70 Assuming an extrapolation such as from Ref ${ }^{74}$ which exceeds the PDG fit by a factor $\sim 10$ at $10^{20} \mathrm{eV}$, a reduction of $X_{\max }$ by $\sim 100 \mathrm{~g} \mathrm{~cm}^{-2}$ is possible for unconverted photons. Already with a small sample of observed photon showers, such a scenario can be distinguished from more "standard" ones. 


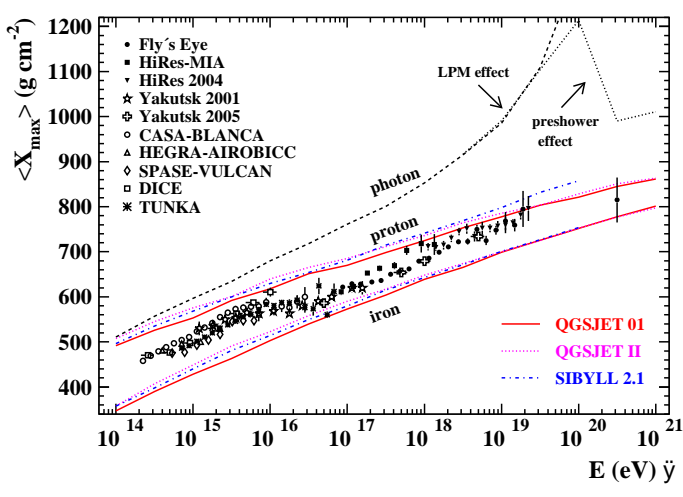

Fig. 5. Average depth of shower maximum $\left\langle X_{\max }\right\rangle$ versus energy simulated for primary photons, protons and iron nuclei. The impact of the LPM and preshower effects on $\left\langle X_{\max }\right\rangle$ is visible. The splitting of the photon line at $\sim 3 \times 10^{19} \mathrm{eV}$ indicates that $\left\langle X_{\max }^{\gamma}\right\rangle$ above these energies depends also on the specific trajectory through the geomagnetic field. For nuclear primaries, calculations for different hadronic interaction models are displayed (SIBYLL 2.167, QGSJET 01, 68 QGSJET II 69). See Ref 78 for references to the experimental data.

\subsection{Features of photon-induced showers}

Shower profiles calculated with the preshower and LPM effects switched on/off are shown in Figure 4 for the conditions of the $3.2 \times 10^{20}$ Fly's Eye event $\frac{75}{\text {. Both }}$ effects compete with each other: the LPM effect increases the average $X_{\max }$ and its event-by-event fluctuations. The preshower effect, in turn, reduces both quantities.

Calculations of average $X_{\max }$ values for different primaries are shown in Figure 5 , The large "elongation rate" (slope $d X_{\max } / d \lg E$ ) for photons leads to $X_{\max }$ values well above those of nuclear primaries already at $10^{17}-10^{18} \mathrm{eV}$. Changes in the elongation rate at $10^{19}-10^{20} \mathrm{eV}$ are due to the LPM and preshower effects.

In addition to $X_{\max }$, showers from photon and nuclear primaries differ in $N_{\mu}$ due to the small photonuclear cross-section. To study the impact of shower fluctuations on photon identification, a scatter plot of $X_{\max }$ versus $N_{\mu}$ is given in Figure 6 for different primaries simulated at $3 \times 10^{19} \mathrm{eV}$. This is an idealized plot as no detector effects are accounted for. It can be seen that shower fluctuations by themselves do not impose major limitations to identify photons. Misidentification rates of $<10^{-3}$ $\left(<10^{-4}\right)$ using $X_{\max }\left(N_{\mu}\right)$ seem possible for protons and are even smaller for nuclei (numbers may depend on the hadron generator used for calculating nuclear primaries). In turn, an unconverted primary photon may hardly be distinguished from a nuclear primary if in one of the first interactions, a photonuclear interaction occurs (probability of order $\sim 1 \%$ ). Contrary to $X_{\max }, N_{\mu}$ does not differ much between converted and unconverted photons 50 Further studies of photon showers can be found, for instance, in Refs. $79|80| 81|82| 83|84| 85|86| 87$.

There are other observables, usually closely related to $X_{\max }$ or $N_{\mu}$ or a combination of both, that could be used to distinguish photons from nuclear primaries. These observables, some of which are specific for a certain detector type and experimental configuration, include the curvature of the shower front and the steepness of the lateral distribution $\frac{87}{}$ of ground particles, the risetime 34 of the detector signal at a certain core distance as well as the signal amplitude by itself. 


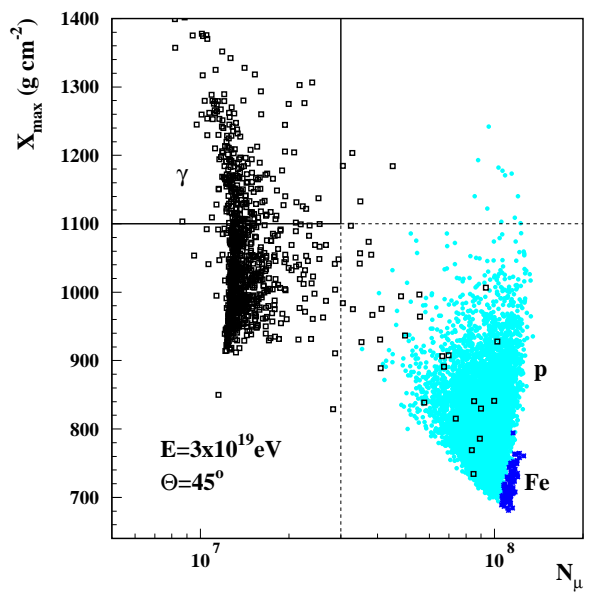

Fig. 6. $X_{\max }$ versus $N_{\mu}$ for different primaries at $3 \times 10^{19} \mathrm{eV}$. Simulations were performed for photons (1000 events), protons (10000 events), and iron nuclei (100 events) with PRESHOWER, CONEX and QGSJET 01. No detector effects are included. The lines illustrate possible cuts to achieve small misidentification rates for photon identification (see text).

\subsection{Detector response to photon-induced showers}

The differences between showers from photons and nuclear primaries can affect the detector acceptance to and the energy reconstruction of UHE photons. As the effects depend on the detector type, analysis approaches appropriate for the experimental apparatus can be developed to minimize uncertainties from the corrections.

Suppose a two-component composition of photons and protons with fluxes $\Phi_{\gamma}(E)$ and $\Phi_{p}(E)$ at energy $E$. The ratio of photons to protons, one measure of the photon fraction, is then $f_{\gamma p}(E)=\Phi_{\gamma}(E) / \Phi_{p}(E)$. Similarly, integral fluxes or fractions, additional primaries, or the photon fraction to the total flux could be considered.

Regarding the detector acceptances $A_{i}(E)$ with $i=\gamma$ or $p$, an acceptance ratio of $\epsilon(E)=A_{\gamma}(E) / A_{p}(E)<1$ affects both the flux and fraction of registered photons. Such a bias can occur for near-vertical photon showers reaching ground before being fully developed. For instance, the aperture of the HiRes-I telescope was found to be $\sim 40 \%$ reduced for photons at $E_{\mathrm{GZK}} \cdot \frac{88}{\mathrm{In}}$ a recent analysis of Auger hybrid data, a minimum zenith angle was introduced to reduce the acceptance bias to photons. 34 Experimental conclusions about the photon flux require knowledge of $A_{\gamma}(E)$ (usually well controlled for arrays using appropriate selection cuts). For conclusions about the photon fraction, knowledge of the relative acceptance $\epsilon(E)$ (possibly easier to calculate for fluorescence telescopes) may be sufficient. The latter approach has been applied in the analysis of Auger hybrid data 34

Reconstructing an energy $E_{\text {rec }}$ from observables that, on average, differ between photon and proton showers of same energy $E$ and assuming proton primaries only, leads to a misreconstruction of photon energies. Selecting events according to $E_{\text {rec }}$ then introduces a shift in energy scales between the primaries: events with the same $E_{\text {rec }}$ refer to protons of energy $E_{\text {rec }}=E$, but to photons of energy $E_{\text {rec }} / g=E / g$ with $g \neq 1$. Hence, the photon flux and fraction actually entering the data sample 
are $\Phi_{\gamma}(E / g) \neq \Phi_{\gamma}(E)$ and $\Phi_{\gamma}(E / g) / \Phi_{p}(E) \neq f_{\gamma p}(E)$. In general, a correction for this shift in energy scales is required.

For the fluorescence technique using the integrated calorimetric shower energy as an observable, $g \sim 1.1$, i.e. the energy scales nearly match and $g>1$. The small difference in energy scales is due to the missing energy correction, which is larger for nuclear primaries compared to the almost purely electromagnetic photon showers ${ }^{89}$ A conservative upper limit to the integral flux or fraction of photons above $E$ may then be possible without correction or assumptions on $\Phi_{\gamma}(E)$ since for $g \sim 1.1 \Rightarrow E / g<E \Rightarrow \int_{E / g} \Phi_{\gamma}\left(E^{\prime}\right) d E^{\prime} \geq \int_{E} \Phi_{\gamma}\left(E^{\prime}\right) d E^{\prime}$. This was used in Ref. 34 .

For arrays when reconstructing the energy from a signal amplitude $S(r)$ measured at ground at core distance $r$, the factor $g$ depends in general on the arrival direction of the event (slant depth to ground changes with zenith, preshower probability depends on zenith and azimuth), its energy (dependence of $X_{\max }$ with energy, see Figure 5), and detector characteristics (e.g. sensitivity to shower muons). Values

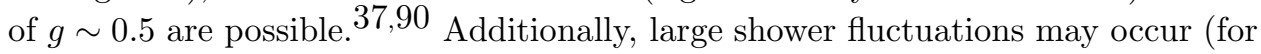
a proposal to reduce the effect of shower fluctuations when reconstructing photon energies, see Ref.91). The (for arrays larger) uncertainty when relating different energy scales may be avoided by analysing the photon flux rather than the fraction, as for the flux, only the photon energy scale is required (and $A_{\gamma}(E)$ is usually well controlled for arrays, see above).

Within the flux level experimentally allowed for photons, the different detector response to photons introduces a systematic uncertainty when reconstructing an energy spectrum. An example of how the high-energy end of the spectrum from first Auger data can change when simply assuming a constant factor two between photon and proton energies is shown in Ref 92 . Detector responses to deep showers in general are discussed in Ref. 93 .

\section{Experimental Searches for UHE Photons}

\subsection{Status}

No photon detection has been reported so far. Upper limits to photons come from different experiments, see also Figure 2 .

Comparing rates of near-vertical showers to inclined ones recorded with the Haverah Park water detectors, upper limits (95\% c.l.) of $48 \%$ above $10^{19} \mathrm{eV}(52$ events) and $50 \%$ above $4 \times 10^{19} \mathrm{eV}$ (10 events) were deduced ${ }^{35|36|}$ From simulations, ground signals were found to be stronger suppressed for photons than for nuclear primaries at large zenith angles. The absence of this suppression in the data gives constrains on the photon contribution. Contrary to other approaches, a feature of the data sample as a whole is analysed here instead of observables in single events.

With muon counters in the AGASA array, the muon density $\rho_{\mu}$ at $1000 \mathrm{~m}$ core distance was measured for part of the events $\frac{37}{}$ A simulation study of the ground signal $\mathrm{S}(600)$ for different primaries showed a possible underestimation of photon energies (e.g. about $30 \%, 50 \%, 20 \%$ at $10^{19} \mathrm{eV}, 10^{19.5} \mathrm{eV},>10^{20} \mathrm{eV}$ ). Assuming a 
mixture of primary photons and protons, limits (95\% c.l.) to the photon fraction were estimated to be $28 \%$ above $10^{19} \mathrm{eV}$ (102 events) and $67 \%$ above $3.2 \times 10^{19} \mathrm{eV}$ (14 events) $! 37$ The data were compared to an overall simulated distribution, i.e. not event-by-event. There seem to be some "photon-like" events (not commented on in the paper): in about five events, the measured $\rho_{\mu}$ is considerably (factor $\sim 4$ or more) below typical values expected for nuclear primaries; in additional four events, the (at least two) muon detectors registered no signal; one event of $E_{\mathrm{rec}} \sim 7.5 \times 10^{19} \mathrm{eV}$ (energy scale for nuclear primaries) was observed with a value $\rho_{\mu}$ about a factor $\sim 2.5$ below an average expected from a data fit.

A comparison to individual events is most interesting at highest energy where every second event could be a photon (cf. Figure 2). Observed event features can be compared to high-statistics photon simulations, and the chance probability of photons to generate such an event can be determined. This way, for the $3.2 \times 10^{20} \mathrm{eV}$ Fly's Eye event ${ }^{75]}$ (see Figure 41) with $X_{\max }^{\text {obs }} \sim 815 \pm 60 \mathrm{~g} \mathrm{~cm}^{-2}$, photon shower profiles (with $X_{\max }^{\gamma} \sim 937 \pm 26 \mathrm{~g} \mathrm{~cm}^{-2}$ ) were found to differ by $\sim 1.5 \sigma$ from the observed profile. ${ }^{7776}$ While a photon origin can not be excluded, profiles from nuclear primaries fit the data better $94 \mid 76$

The method of comparing individual events was also applied in Ref. 38 to the six highest energy AGASA events with observed $\rho_{\mu}$. The energy scale for photons and the uncertainties of reconstructed energy and $\rho_{\mu}$ were taken according to AGASA findings in Ref 37 . For all six events, the muon densities from photons are (factors $2-7$ ) below the observed ones. Photon predictions would be even more discrepant to data if smaller energies (see below) were assumed. A statistical approach for deriving limits from a small number of events was developed which accounts for shower features changing for each event and for non-Gaussian shower fluctuations. An upper limit of $67 \%$ (95\% c.l.) above $1.25 \times 10^{20} \mathrm{eV}$ was derived. Though not ruling out non-acceleration models, these scenarios appear to have problems to consistently explain the AGASA data (no flux suppression and no photon dominance).

A method for comparing measured and simulated shower observables such as

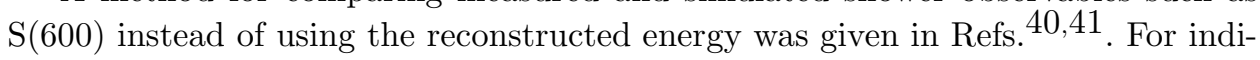
vidual events, the corresponding photon energies are found by requiring the simulated $\mathrm{S}(600)$ to fit the observed ones and properly weighting the events. This way, shower and detector fluctuations can more directly be accounted for. Detailed information about the detector is needed $40 \mid 41$

Applied to AGASA data, using response functions for $\mathrm{S}(600)$ and information on the muon detector response from Ref 37 , photon energies of the six highestenergy events were found in Ref ${ }^{40}$ to be (up to factor $\sim 2$ ) below those expected from Ref. 37 . There appear to be differences between the findings of Refs 40 and 37 in the expected $\mathrm{S}(600)$ for fixed energy which may be connected to the use of different simulation codes. Combining six AGASA $^{37}$ and four Yakutsk 95 events with $E_{\text {rec }}>8 \times 10^{19} \mathrm{eV}$ (energy scale for nuclear primaries), a $36 \%$ limit above

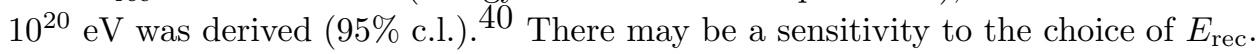


Reducing $E_{\text {rec }}$, a more "photon-like" AGASA event (see above) enters the sample. For a discussion about combining data from these two experiments which appear to have systematic differences in the reconstructed flux spectra, see e.g. Ref!33.

Using the same method of Ref. $40[41$ and investigating Yakutsk data only, preliminary (not yet published) limits of $12 \%$ above $2 \times 10^{19} \mathrm{eV}$ (50 events) and $22 \%$ above $4 \times 10^{19} \mathrm{eV}$ (95\% c.l.) were obtained ${ }^{39}$ In two events no muon signal was registered, another event was classified as muon-poor.

All these limits refer to the fraction of photons and come from ground arrays. The fluorescence technique provides important cross-checks due to smaller uncertainties from different energy scales of the primaries (Section 3.3). Using $X_{\max }$ from the direct observation of the shower profile with fluorescence telescopes in hybrid events (i.e. registered by ground and fluorescence detectors) a limit of $16 \%$ (95\% c.l.) above $10^{19} \mathrm{eV}$ (29 events) was obtained from first data taken at the Auger Observatory $\frac{34}{34}$ An approach was developed such that the limit does not rely on assumptions about input spectra or composition. A $\sim 2 \times 10^{20} \mathrm{eV}$ event registered with $X_{\max }^{\text {obs }} \sim 821 \pm$ $36 \mathrm{~g} \mathrm{~cm}^{-2}$ differs by $\sim 3 \sigma$ from the photon hypothesis $\left(X_{\max }^{\gamma} \sim 948 \pm 27 \mathrm{~g} \mathrm{~cm}^{-2}\right)$.

Steps were taken to analyse the HiRes stereo data set of about 50 events above $4 \times 10^{19} \mathrm{eV} \underline{\underline{96}}$ No results were reported so far.

In conclusion, current data from ground and fluorescence detectors do not indicate a large (above $10^{19} \mathrm{eV}$ ) or dominant (above $10^{20} \mathrm{eV}$ ) photon contribution. Non-acceleration models are constrained by existing limits though not ruled out as an explanation of UHE cosmic rays (see also discussion in Ref. 33 ).

\subsection{Prospects}

The $16 \%$ limit from the Auger hybrid data is based on 29 events. The sensitivity can be improved by a factor $\sim 3$ or more with data accumulated until 2008/2009 34 Also energies below $10^{19} \mathrm{eV}$ can be reached with the hybrid technique.

For an overall estimate of future sensitivities it is instructive to consider the lowest theoretical fraction $F_{\gamma}^{\min }$ that can be excluded with $n$ events. With $\alpha$ being the confidence level of rejection, $F_{\gamma}^{\min }$ is

$$
F_{\gamma}^{\min }(n)=1-(1-\alpha)^{1 / n} \approx 3 / n \text { for } \alpha=0.95, n \gg 1 .
$$

A limit derived from data can exceed $F_{\gamma}^{\min }$ (i.e. be weaker) depending on the discrimination power of the specific observables (possibly limited by shower fluctuations and detector characteristics), efficiency corrections, and the actual number of photons in the data sample. For the example of the Auger hybrid limit based on 29 events, $F_{\gamma}^{\min }(29) \sim 10 \%$. The final limit of $16 \%$, which includes a $\sim 20 \%$ efficiency correction, corresponds to the theoretical limit for 17 events $\left(F_{\gamma}^{\min }(17) \sim 16 \%\right)$. Thus, compared to the 29 events in the data set, the $16 \%$ limit corresponds to an effective number of events that is smaller by a factor $r \sim 29 / 17 \sim 1.7$.

To estimate the sensitivity to photons of the large Auger array, we assume a factor of $r \sim 2.4$, i.e. larger (less discriminative events and/or larger efficiency 


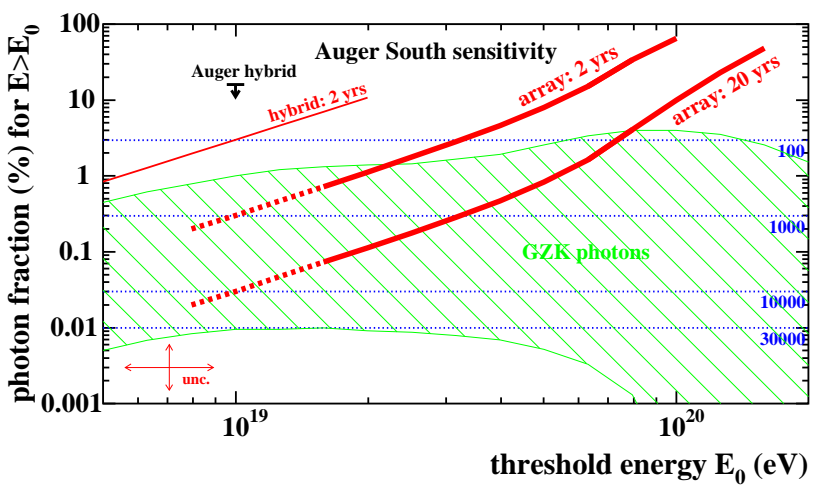

Fig. 7. Fraction of photons in the integral cosmic-ray flux as a function of the threshold energy (see also Fig. 2). The predictions 21 (labeled "GZK photons") for primary nucleon sources follow a spectrum with flux suppression above $E_{\mathrm{GZK}}$. For reference, the current limit from Auger hybrid data, obtained with 29 events, is shown. The estimated sensitivity (see text) of the array of the southern Auger Observatory is shown for two and twenty years of operation for a spectrum with flux suppression. Also shown is the estimated sensitivity using hybrid data, which are less numerous due to the $\sim 10 \%$ duty cycle but reach to smaller energy. The uncertainty of the sensitivity estimates is indicated at the lower left corner. At around $10^{19} \mathrm{eV}$ (dashed lines), additional threshold effects may become increasingly important for the array. If complemented by an extended northern array, a sensitivity level of below $0.1 \%$ can be reached within a few years of full operation.

correction) than the factor reached in the first Auger hybrid limit. Corresponding numbers from other event-by-event photon searches using AGASA and Yakutsk data

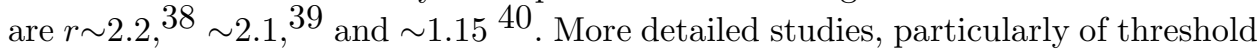
effects, are needed. However, the uncertainty of this estimate (factor $\sim 2$ ) seems well below that of current theoretical predictions of UHE photon fluxes. Using for $n\left(E>E_{0}\right)$ the Auger energy spectrum $\frac{90}{9 i v e s}$ the sensitivity estimates $F\left(E>E_{0}\right)$ shown in Figures 2 and 7 by calculating $F=F_{\gamma}^{\min }\left(n^{\prime}\right) \sim 3 r / n$ where $n^{\prime}=n / r$ denotes the effective number of events ${ }^{a}$ The curve $F\left(E_{0}\right)$ indicates the upper limit that can be set at $95 \%$ c.l. if there were no photons in the data. The curve represents also (since $F\left(E_{0}\right) \sim 3 r / n$ ) the ratio of $3 r \simeq 7$ to $n$ events: if it is possible to identify 7 photons out of $n\left(E_{0}\right)$ events, $F\left(E_{0}\right)$ gives the observed photon fraction.

A crucial test of non-acceleration models is possible with the southern Auger array even if the flux is suppressed above $E_{\mathrm{GZK}}$ (Figure 2). A large exposure is required to reach sensitivities of $\sim 0.1 \%$ or below, i.e. to possibly observe GZK photons expected from UHE nucleon sources (Figure 7). Such sensitivity levels may be reached on acceptable time scales if the southern Auger Observatory is complemented by an expanded (e.g. factor 3-4 larger) northern site.

a With the integral number of events $n\left(E>E_{0}\right)$, from Eq. (5) it follows $F_{\gamma}^{\text {min }} \propto E_{0}^{\beta-1}$ for a power law spectrum of differential index $\beta$ ( $\beta \sim 2.84$ from Ref. 90), i.e. a straight line of slope $\beta-1$ in double-logarithmic scales. Zenith angles were restricted to $30-60^{\circ}$ for the estimate of the sensitivity. 
The Telescope Array 98 of scintillators, under construction in Utah, will cover a $\sim 760 \mathrm{~km}^{2}$ area ( $\sim 25 \%$ of the southern Auger array) overviewed by fluorescence telescopes. Space missions such as the proposed EUSO 99 and OWL ${ }^{100}$ projects may offer a significant increase of the experimental exposure to cosmic rays at highest energy.

\section{Possible Impact of UHE Photon Searches}

Photons, as the gauge bosons of the electromagnetic force, at such enormous energy can be regarded as unique messengers and probes of extreme and, possibly, new physics. Implications are related to the production of photons, their propagation, and interactions at the Earth. Many aspects of the following, incomplete list of possible impacts of UHE photon searches and connections to other research subjects require more study.

Large UHE photon fluxes are a smoking gun for current non-acceleration models. Stringent photon limits give parameter constraints such as a lower limit on the lifetime of relic SHDM particles $\underline{33}$

Findings on photons are needed to reduce corresponding systematics in other air shower studies, such as for the energy spectrum (Section 3.3) or when trying to constrain interaction parameters such as the proton-air cross-section 101102 from showers.

UHE photons may be helpful for diagnostics of sources accelerating nuclear primaries, as the photon fluxes from UHE hadron interactions are expected to be connected with source features such as type of primary, injection spectrum, possible beam dump at the source, or source distribution (see also Ref. 21 ).

UHE photons point back to the location of their production. Possibly, the arrival directions of photons may correlate better with the source direction than those of charged primaries. There may be an enhanced UHE photon flux from the galactic center region depending on the spectra of nuclear primaries. 28 In certain SHDM scenarios, an enhanced flux of $\sim 10^{18} \mathrm{eV}$ photons from the galactic center is possible without a higher-energy counterpart 103

Propagation features of UHE photons are sensitive to the $\mathrm{MHz}$ radio background 20 The photon flux at Earth is also sensitive to extragalactic magnetic fields 104

Already a small sample of photon-induced showers may provide relatively clean probes of aspects of QED and QCD at ultra-high energy via the preshower process and photonuclear interactions (Section 3.1).

Certain implications will be most powerful in combination with other results from shower observation (spectrum, anisotropy, nuclear composition) and, probably, with results using other messenger particles (UHE neutrinos, lower energy photons). Parallel to UHE photons, UHE neutrinos are usually produced, see Eq. (1). Due to the much longer mean free path of UHE neutrinos, searches for UHE photons and UHE neutrinos complement each other, with UHE photons (neutrinos) testing 
more local (distant) production sites. The disappearance of UHE photons during propagation is accompanied by an appearance of $\mathrm{GeV}-\mathrm{TeV}$ photons, cf. Eq. (2) and Figure 1 (see also Ref. 105 and references therein). Similar to (but independent of) UHE neutrinos, these $\mathrm{GeV}-\mathrm{TeV}$ photons allow a test of more distant production sites. The close relation between different messengers is reflected by the fact that constraints on non-acceleration models also come from UHE neutrino and $\mathrm{GeV}$ photon data, see e.g. Refs.19|106. The full information could be exploited by multimessenger observations. For instance, for known sources of UHE cosmic rays, fluxes of UHE photons much below the expected level could indicate certain new physics.

There are several connections to Lorentz invariance violation. $107 / 108$ The production of GZK photons can be affected as well as interactions of photons during propagation and when initiating a cascade at the Earth. Particularly, photon conversion (interaction with background fields or preshower process) may be suppressed.

It is interesting to check whether UHE photon propagation could be affected by the presence of axions or scalar bosons. Formal requirements for photon conversion regarding photon energy and magnetic field strength, appear to be fulfilled $109[110$ Photon conversions to non-electromagnetic channels may differ from the standard QED process of Eq. (2) due to an absence of an electromagnetic sub-cascade.

UHE photon propagation can be modified in certain models of brane worlds, 111 quantum gravity theory, ${ }^{112[113}$ or spacetime foam $114 \mid 115$. For instance, depending on fundamental length scales significant scattering of photons on structures (defects) in spacetime foam can occur. In turn, constraints may be derived when actually observing UHE photons, even with one gold-plated event only! 114]115

\section{Conclusions}

UHE photons are tracers of highest-energy processes and new physics. Showers initiated by such photons can well be distinguished from those by nuclear primaries. When complemented by a large northern site, the Pierre Auger Observatory is expected to be sensitive to photon fractions of $0.1 \%$ and below and can realistically aim at photon observations. The search for UHE photons contributes to multimessenger observations of the universe. A key characteristic for the progress in astrophysics is to expand to photon wavelengths beyond the optical; the observation of UHE photons would bring this to the highest end.

\section{Acknowledgments}

We thank D. Barnhill, J. Bellido, V. Berezinsky, P. Billoir, B. Dawson, K. Eitel, R. Engel, E. Gabrielli, D. Góra, D. Gorbunov, J.-C. Hamilton, M. Healy, D. Heck, M. Kachelrieß, K.-H. Kampert, F.R. Klinkhamer, S. Ostapchenko, J. Pȩkala, M. Roth, C. Roucelle, S. Sarkar, D. Semikoz, K. Shinozaki, G. Sigl, P. Sommers, M. Strikman, S. Troitsky, M. Unger, S. Vorobiov, A.A. Watson, B. Wilczyńska, H. Wilczyński, and all members of the Auger "photon group" for many useful discussions. The simulations and plot routines for Figure 5 were kindly provided 
by D. Heck. This work was partially supported by the Polish Ministry of Science and Higher Education (Grant N202 090 31/0623) and by the German Ministry for Research and Education (Grant 05 CU5PX1/6). One of the authors (MR) kindly acknowledges support from the Alexander von Humboldt foundation. Preprint of a brief review submitted for consideration in Modern Physics Letters A (copyright World Scientific Publishing Company, 2007, http://www.worldscientific.com.sg).

\section{References}

1. See, for instance, T.C. Weekes, arXiv:astro-ph/0606130, and references therein.

2. Pierre Auger Collaboration, Nucl. Instr. Meth. A 523, 50 (2004).

3. M. Nagano, A.A. Watson, Rev. Mod. Phys. 72, 689 (2000).

4. L. Anchordoqui et al., Int. J. Mod. Phys. A 18, 2229 (2003).

5. "Ultimate energy particles in the Universe", eds. M. Boratav and G. Sigl, C.R. Physique 5, Elsevier, Paris (2004).

6. J. Cronin, Nucl. Phys. B, Proc. Suppl. 138, 465 (2005).

7. F. Halzen, D. Hooper, Rep. Prog. Phys. 65, 1025 (2002).

8. A.B. McDonald et al., Rev. Sci. Instrum. 75, 293 (2004).

9. P. Bhattacharjee, G. Sigl, Phys. Rep. 327, 109 (2000).

10. C.T. Hill, Nucl. Phys. B 224, 469 (1983).

11. M.B. Hindmarsh, T.W.B. Kibble, Rep. Prog. Phys. 58, 477 (1995).

12. V. Berezinsky, M. Kachelrieß, A. Vilenkin, Phys. Rev. Lett. 79, 4302 (1997).

13. M. Birkel, S. Sarkar, Astropart. Phys. 9, 297 (1998).

14. V.A. Kuzmin, V.A. Rubakov, Phys. Atom. Nucl. 61, 1028 (1998).

15. P. Blasi, R. Dick, E.W. Kolb, Astropart. Phys. 18, 57 (2002).

16. Z. Fodor, S.D. Katz, Phys. Rev. Lett. 86, 3224 (2001).

17. S. Sarkar, R. Toldra, Nucl. Phys. B 621, 495 (2002).

18. C. Barbot, M. Drees, Astropart. Phys. 20, 5 (2003).

19. R. Aloisio, V. Berezinsky, M. Kachelrieß, Phys. Rev. D 69, 094023 (2004).

20. S. Sarkar, Acta Phys. Polon. B35, 351 (2004).

21. G. Gelmini, O.E. Kalashev, D.V. Semikoz, arXiv:astro-ph/0506128.

22. J. Ellis, V. Mayes, D.V. Nanopoulos, Phys. Rev. D 74, 115003 (2006).

23. T.J. Weiler, Phys. Rev. Lett. 49, 234 (1982).

24. T.J. Weiler, Astropart. Phys. 11, 303 (1999).

25. D. Fargion, B. Mele, A. Salis, Astrophys. J. 517, 725 (1999).

26. K. Greisen, Phys. Rev. Lett. 16, 748 (1966).

27. G.T. Zatsepin, V.A. Kuzmin, JETP Lett. 4, 78 (1966).

28. A. Kusenko, J. Schissel, F.W. Stecker, Astropart. Phys. 25, 242 (2006).

29. S. Lee, Phys. Rev. D 58, 043004 (1998).

30. D. Hooper, S. Sarkar, A.M. Taylor, Astropart. Phys. 27 (2007) 199.

31. R.U. Abbasi et al., Phys. Lett. B 619, 271 (2005).

32. M. Takeda et al., Astropart. Phys. 19, 447 (2003).

33. R. Aloisio, V. Berezinsky, M. Kachelrieß, Phys. Rev. D 74, 023516 (2006).

34. Pierre Auger Collaboration, Astropart. Phys. 27, 155 (2007).

35. M. Ave et al., Phys. Rev. Lett. 85, 2244 (2000).

36. Phys. Rev. D 65, 063007 (2002).

37. K. Shinozaki et al., Astrophys. J. 571, L117 (2002).

38. M. Risse et al., Phys. Rev. Lett. 95, 171102 (2005).

39. A.V. Glushkov et al., arXiv:astro-ph/0701245 1 ].

40. G.I. Rubtsov et al., Phys. Rev. D 73, 063009 (2006). 
41. D.S. Gorbunov, G.I. Rubtsov, S.V. Troitsky, arXiv:astro-ph/0606442 .

42. G. Sigl, arXiv:astro-ph/0612240.

43. T. Erber, Rev. Mod. Phys. 38, 626 (1966) (see Refs. $52|63| 64$ for a caveat related to Table VI of the paper);

44. V.H. Bayer, B.M. Katkov, V.S. Fadin, Radiation of Relativistic Electrons (in Russian), Atomizdat, Moscow (1973);

45. V.B. Berestetskii et al., Quantum Electrodynamics, Pergamon Press, 2nd edition (1982);

46. A.A. Sokolov, I.M. Ternov, Radiation from Relativistic Electrons, Springer Verlag (1986);

47. M.G. Baring, A\&A 225, 260 (1989);

48. U.I. Uggerhøj, Nucl. Phys. B (Proc. Suppl.) 122, 357 (2003).

49. J. K. Daugherty, A. K. Harding, Astrophys. J. 273, 761 (1983).

50. P. Homola et al., Astropart. Phys. 27 (2007) 174.

51. M. Coraddu et al., arXiv:hep-ph/0210107.

52. P. Homola et al., Comput. Phys. Commun. 173, 71 (2005).

53. D. Heck et al., Reports FZKA 6019 \& 6097, Forschungszentrum Karlsruhe (1998).

54. T. Bergmann et al., Astropart. Phys. 26, 420 (2006).

55. D. Badagnani, S.J. Sciutto, Proc. $29^{\text {th }}$ Intern. Cosmic Ray Conf., Pune, 9, 1 (2005).

56. S.J. Sciutto, arXiv:astro-ph/9911331.

57. National Geophysical Data Center, USA, http://www.ngdc.noaa.gov.

58. H.A. Bethe, W. Heitler, Proc. Roy. Soc., A146 83 (1934).

59. W.M. Yao et al., J. Phys. G: Nucl. Part. Phys. 33, 1 (2006).

60. L.D. Landau, I.Ya. Pomeranchuk, Dokl. Akad. Nauk SSSR 92, 535 (1953).

61. L.D. Landau, I.Ya. Pomeranchuk, Dokl. Akad. Nauk SSSR 92, 735 (1953).

62. A.B. Migdal, Phys. Rev. 103, 1811 (1956).

63. S. Klein, Rev. Mod. Phys. 71, 1501 (1999).

64. S. Klein, Rad. Phys. Chem. 75, 696 (2006).

65. H.D. Hansen et al., Phys. Rev. Lett. 91, 014801 (2003).

66. A.N. Cillis et al., Phys. Rev. D 59, 113012 (1997).

67. R. Engel et al., Proc. $26^{\text {th }}$ Intern. Cosmic Ray Conf., Salt Lake City, 415 (1999).

68. N.N. Kalmykov, S.S. Ostapchenko, A.I. Pavlov, Nucl. Phys. B (Proc. Suppl.) 52, 17 (1997).

69. S. Ostapchenko, Nucl. Phys. B (Proc. Suppl.) 151, 143 (2006).

70. M. Risse et al., Czech. J. Phys. 56, A327 (2006).

71. T.C. Rogers, M.I. Strikman, J. Phys. G: Nucl. Part. Phys. 32, 2041 (2006).

72. S. Eidelmann et al., Phys. Lett. B 592, 1 (2004).

73. J.R. Cudell et al., Phys. Rev. D 65, 074024 (2002).

74. A. Donnachie, P. Landshoff, Phys. Lett. B 518, 63 (2001).

75. D.J. Bird et al., Astrophys. J. 441, 144 (1995).

76. M. Risse et al., Astropart. Phys. 21, 479 (2004).

77. F. Halzen, arXiv:astro-ph/0302489.

78. J. Knapp et al., Astropart. Phys. 19, 77 (2003).

79. B. McBreen, C.J. Lambert, Phys. Rev. D 24, 2536 (1981).

80. F.A. Aharonian, B.L. Kanevsky, V.V. Vardanian, Astrophys. Space Sci. 167, 111 (1990).

81. H.P. Vankov, P.V. Stavrev, Phys. Lett. B 266, 178 (1991).

82. T. Stanev, H.P. Vankov, Phys. Rev. D 55, 1365 (1997).

83. A.V. Plyasheshnikov, F.A. Aharonian, J. Phys. G 238, 267 (2002).

84. J.N. Capdevielle, C. Le Gall, Kh.N. Sanosyan, Astropart. Phys. 13, 259, (2000). 
85. W. Bednarek, New Astronomy 7, 471 (2002).

86. H.P. Vankov, N. Inoue, K. Shinozaki, Phys. Rev. D 67, 043002 (2003).

87. X. Bertou, P. Billoir, S. Dagoret-Campagne, Astropart. Phys. 14, 121 (2000).

88. V. de Souza, G. Medina-Tanco, J.A. Ortiz, Phys. Rev. D 72, 103009 (2005).

89. T. Pierog et al., Proc. $29^{\text {th }}$ Intern. Cosmic Ray Conf., Pune, 7, 103 (2005).

90. P. Sommers for the Auger Collaboration, Proc. $29^{\text {th }}$ Intern. Cosmic Ray Conf., Pune, 7, 387 (2005).

91. P. Billoir, C. Roucelle, J.-C. Hamilton, arXiv:astro-ph/0701583.

92. N. Busca, D. Hooper, E.W. Kolb, Phys. Rev. D 73, 123001 (2006).

93. A.S. Chou, Phys. Rev. D 74, 103001 (2006).

94. F. Halzen et al., Astropart. Phys. 3, 151 (1995).

95. V. Egorova et al., Nucl. Phys. B (Proc. Suppl.) 136, 3 (2004).

96. HiRes Collaboration, Proc. $29^{\text {th }}$ Intern. Cosmic Ray Conf., Pune 7, 373 (2005).

97. With the integral number of events $n\left(E>E_{0}\right.$ ), from Eq. (5) it follows $F_{\gamma}^{\text {min }} \propto E_{0}^{\beta-1}$ for a power law spectrum of differential index $\beta,(\beta \sim 2.84$ from Ref. 90$)$, i.e. a straight line of slope $\beta-1$ in double-logarithmic scales. Zenith angles were restricted to $30-60^{\circ}$ for the estimate of the sensitivity.

98. M. Fukushima et al., Prog. Theor. Phys. Suppl. 151, 206 (2003).

99. http://www.euso-mission.org

100. http://owl.gsfc.nasa.gov

101. K. Belov et al., Nucl. Phys. B (Proc. Suppl.) 151, 197 (2006).

102. R. Ulrich et al., arXiv:astro-ph/0612205.

103. V. Berezinsky, private communication (2005).

104. S. Lee, A.V. Olinto, G. Sigl, Astrophys. J. 455, L21 (1995).

105. E. Armengaud, G. Sigl, F. Miniati, Phys. Rev. D 73, 083008 (2006).

106. D.V. Semikoz, G. Sigl, JCAP 0404, 003 (2004).

107. T. Jacobson, S. Liberati, D. Mattingly, Annals Phys. 321, 150 (2006).

108. B. Altschul, arXiv:hep-ph/0610324.

109. E. Gabrielli, K. Huitu, S. Roy, Phys. Rev. D 74, 073002 (2006).

110. E. Gabrielli, private communication (2006).

111. M. Gogberashvili, A.S. Sakharov, E.K.G. Sarkisyan, Phys. Lett. B 644, 179 (2007).

112. G. Amelino-Camelia et al., Nature 393, 763 (1998).

113. R. Gambini, J. Pullin, Phys. Rev. D 59, 124021 (1999).

114. S. Bernadotte, F.R. Klinkhamer, Phys. Rev. D 75, 024028 (2007).

115. F.R. Klinkhamer, private communication (2007) 